\title{
A Review on FPGA Implementation of Edge Detection Algorithms
}

\author{
Avinash G. Mahalle \\ M. Tech Scholar, Government College of Engineering, Amravati
}

\begin{abstract}
Edge detection is a fundamental tool in the field of image processing. Edge indicates sudden change in the intensity level of image pixels. By detecting edges in the image, one can preserve its features and eliminate useless information. In the recent years, especially in the field of Computer Vision, edge detection has been emerged out as a key technique for image processing. There are various gradient based edge detection algorithms such as Robert, Prewitt, Sobel, Canny which can be used for this purpose. This paper reviews all these gradient based edge detection techniques and provides comparative analysis. MATLAB/Simulink is used as a simulation tool. System is designed by configuring ISE Design suit with MATLAB. Hardware Description Language (HDL) is generated using Xilinx System Generator. HDL code is synthesized and implemented using Field Programmable Gate Array (FPGA).
\end{abstract}

Keywords: Image Processing, Edge Detection, MATLAB/Simulink, Xilinx System Generator (XSG), Field Programmable Gate Array (FPGA)

\section{Introduction}

Digital image consists of set of pixels with certain value of intensity. Edge can be characterized by sudden change in this intensity levels [1]. In order to identify these edges from an image various edge detection algorithms have been proposed. All these algorithms are mainly categorized in two groups: Gradient based and Laplacian based. The detailed classification of gradient based edge detectors is given in [2]. Classical gradient based edge detector operators such as Robert, Prewitt, Sobel are simple to design and very effective in real time image processing. But these are very sensitive to Noise. The noise in the image occurred due to light variations, camera electronics, surface reflectance and lens. Image smoothening is done to eliminate noise content in the digital image. Further these classical operators has poor accuracy due to missing true edges, high error rate and thick edges. On the other hand, Canny algorithm [3] provides high performance edge detection as compared to classical operators. Canny algorithms consists of additional blocks such as Non-Maximal Suppression (NMS), Hysteresis thresholding which provides optimal accuracy. At the same time canny algorithm becomes computationally more complex than that of classical operators. This increases its latency and decreases its throughput [4].

Implementing design of different edge detection algorithms uses reconfigurable devices such as FPGA. Main advantages of FPGA is pipelining and parallel processing which makes complex design executable with low latency and high throughput [5]. Hardware Description Language (HDL) coding is required to implement complex algorithms on FPGA. Coding in HDL (VHDL/Verilog) languages can be more tedious job as we have to write code in that particular language [6]. ISE Design Suit 14.4 provides a solution to this difficulty. MATLAB R2013a when configured with ISE Design Suit, required algorithm can be designed with XSG blocks. VHDL code for designed algorithm is generated using XSG. This VHDL code is then synthesized and programming file (.bit file) is generated. Implementing our design on reconfigurable hardware such as FPGA, minimizes time-to-market, enables rapid prototyping of complex algorithm and simplifies debugging and verification [7].

The rest of the paper is organized as follows. Section 2 gives a brief idea about edge detection, its classification and various classical gradient operators. Section 3 discusses Canny algorithm in detail. Section 4 provides System Requirements for proposed approach. Section 5 discusses Simulation and Synthesis. Section 6 deals with flow of Implementation of Edge detection algorithm. Section 7 gives Conclusion.

\section{Edge Detection}

Abrupt change in the intensity values occur at boundary between two regions in image. Background pixels of image has similar and low intensity values. In various applications such as medicine, space exploration, surveillance, authentic automated industry inspection etc., useful information is characterized by edges whereas background portion of images can be eliminated. Fig.1 shows classification of various edge detection techniques.

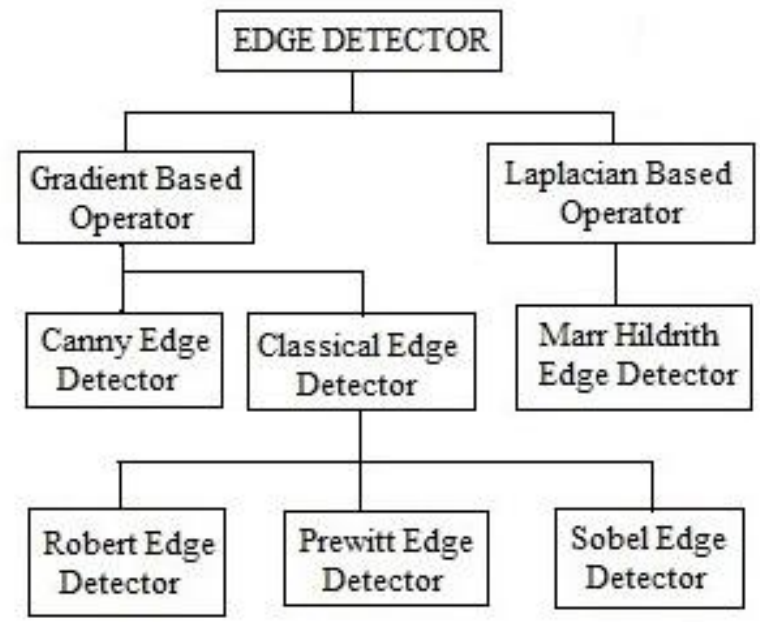

Figure 1: Classification of Edge Detection Techniques

\section{Volume 6 Issue 1, January 2017}




\section{International Journal of Science and Research (IJSR) \\ ISSN (Online): 2319-7064}

Index Copernicus Value (2015): 78.96 | Impact Factor (2015): 6.391

In this paper, gradient based edge detection techniques has been discussed. Rate of change in image pixel intensities is detected by differentiation. Respective masks of various operators are convolved with given gray image.

\section{A. Robert operator:}

Image is set of pixels and can be expressed as $i\left(x_{x} y\right)$, where, $(x, y)$ indicates coordinates and value of function indicates intensity value of image pixel at particular coordinates. Magnitude and orientation of any pixel is calculated as follows:

Magnitude, $\operatorname{mag}(x, y)=\sqrt{g x^{2}+g y^{2}} \ldots \ldots$ (i)

Orientation, $\theta(x, y)=\tan ^{-1}(g y / g x)$

where, $g x=$ change along $\mathrm{x}$-Direction

gy $=$ change along $\mathrm{y}$-Direction

Robert operator is [2×2] mask $f\left(x_{v} y\right)$, which is convolved with image portion. Final result is nothing but gradient $g(x, y)$ of given image region.

\begin{tabular}{|l|l|}
\hline P1 & P2 \\
\hline P3 & P4 \\
\hline
\end{tabular}

Region of Image

\begin{tabular}{|l|l|}
\hline-1 & 0 \\
\hline 0 & 1 \\
\hline
\end{tabular}

\begin{tabular}{|l|l|}
\hline 0 & -1 \\
\hline 1 & 0 \\
\hline
\end{tabular}

Robert operator masks $f x_{s} f y$

$$
\begin{aligned}
& g x=(p 4-p 1) \\
& g y=(p 3-p 2) \ldots(1) \\
& \ldots \ldots(2)
\end{aligned}
$$

\section{B. Prewitt operator:}

It is another gradient based classical operator which uses concept of central differences. It has $[3 \times 3]$ masks $f x$ and $f y$ as shown below:

\begin{tabular}{|l|l|l|}
\hline P1 & P2 & P3 \\
\hline P4 & P5 & P6 \\
\hline P7 & P8 & P9 \\
\hline
\end{tabular}

Region of Image

\begin{tabular}{|l|l|l|}
\hline-1 & 0 & 1 \\
\hline-1 & 0 & 1 \\
\hline-1 & 0 & 1 \\
\hline
\end{tabular}

\begin{tabular}{|c|c|c|}
\hline-1 & -1 & -1 \\
\hline 0 & 0 & 0 \\
\hline 1 & 1 & 1 \\
\hline
\end{tabular}

Prewitt operator masks $f x_{v} f y$

$$
\begin{aligned}
& g x=(p 3-p 1)+(p 6-p 4)+(p 9-p 7) \\
& 9 y=(p 7-p 1)+(p 8-p 2)+(p 9-p 3)
\end{aligned}
$$

\section{Sobel operator:}

It is similar to prewitt operator except weighted central difference is used for one row. Both horizontal and vertical masks are shown:

\begin{tabular}{|l|l|l|}
\hline P1 & P2 & P3 \\
\hline P4 & P5 & P6 \\
\hline P7 & P8 & P9 \\
\hline
\end{tabular}

Region of Image

\begin{tabular}{|c|c|c|}
\hline-1 & 0 & 1 \\
\hline-2 & 0 & 2 \\
\hline-1 & 0 & 1 \\
\hline
\end{tabular}

\begin{tabular}{|c|c|c|}
\hline-1 & -2 & -1 \\
\hline 0 & 0 & 0 \\
\hline 1 & 2 & 1 \\
\hline
\end{tabular}

Sobel operator masks $f x_{x} f y$

$$
\begin{aligned}
& g x=(p 3-p 1)+2(p 6-p 4)+(p 9-p 7) \\
& 9 y=(p 7-p 1)+2(p 8-p 2)+(p 9-p 3)
\end{aligned}
$$

\section{Canny Algorithm}

In 1983, J. F. Canny proposed this method in order to yield high performance of edge detection tools [3]. The main disadvantage of gradient based classical operator is that they are sensitive to noise. Finding gradient is also known as differentiating. Differentiator allows high frequency components which includes noise as well. Also, many edge details are not detected by these operators. Canny algorithm eliminates these disadvantages by providing noise immune high performance edge detection. Three main principles on which Canny algorithm works are low error rate, localization of edge points and single response for single edge [8].

Compared to classical operators Canny possesses additional blocks such as Non-Maximal Suppression, Hysteresis Thresholding etc. (as shown in Fig. 2). Thus, hardware complexity of Canny edge detector is higher than that of classical operators. Following steps are performed in Canny Edge Detection method:

1)Smoothening: Gaussian filter is used in this block to remove noise content present in digital image.

2)Finding gradient magnitude and direction: Approximation methods [4] are used.

3)Non-Maximum Suppression: Only strong edges are detected whereas others are suppressed. Provides thin edge line.

4)Hysteresis Thresholding: It eliminates streaking effect in the output image by applying two thresholds.

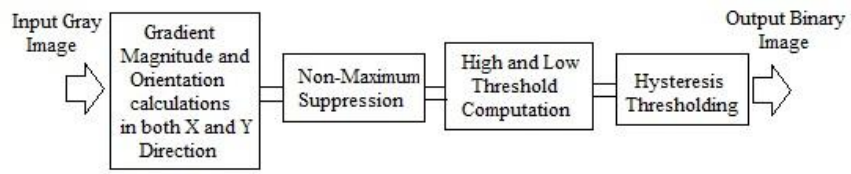

Figure 2: Block Diagram of Canny Algorithm

\section{System Requirements}

MATLAB provides a common environment to Simulink [9] and ISE Design Suit. Fig.3 shows System Generator for MATLAB configurator.

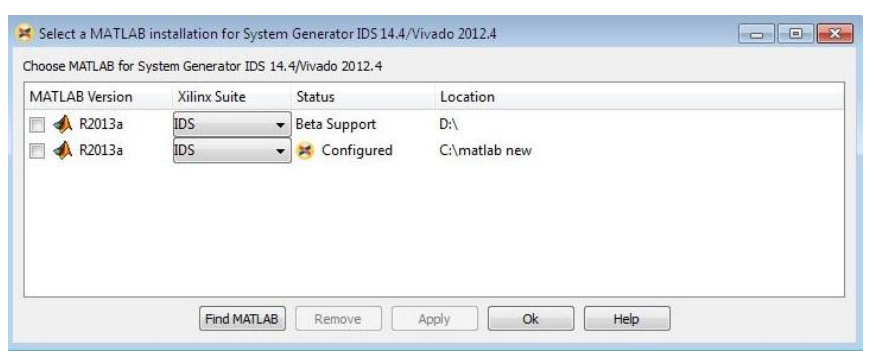

Figure 3: Configuring MATLAB with System Generator

Simulink is a software package for modelling, simulating and analyzing dynamical systems. It provides a graphical user interface for building models as block diagrams. Simulink includes a comprehensive block, library of sinks, sources, linear and nonlinear components and connectors. It can also customize and create the own blocks. Simulink model is synthesized by using Xilinx System Generator [10] Block 


\section{International Journal of Science and Research (IJSR) \\ ISSN (Online): 2319-7064}

Index Copernicus Value (2015): 78.96 | Impact Factor (2015): 6.391

sets. XSG then synthesizes required design and HDL code is generated (as shown in Fig. 4).

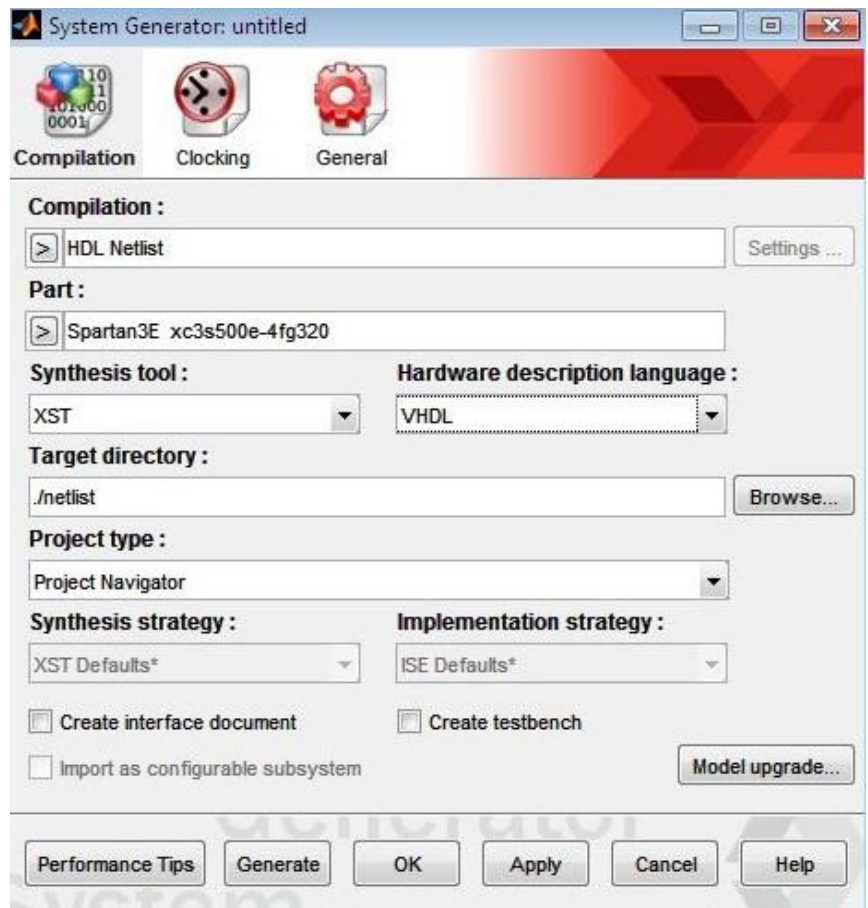

Figure 4: Xilinx System Generator Window

This code can be further simulated and synthesized in ISE 14.4 Design Suit and programming file is generated which can be implemented on Xilinx FPGA.

\section{Simulation and Synthesis}

ISim (ISE Simulator) is an inbuilt simulator for ISE design suit. Modelsim is another simulation tool. Synthesis summary report consists of information related resources used in the implementing design. Figures 5, 6, 7 are obtained from MATLAB code which uses predefined edge function. Accuracy of Robert is the lowest whereas Canny provides optimal edges of the original image. Input to the system is gray image and output is obtained in the form of binary image.

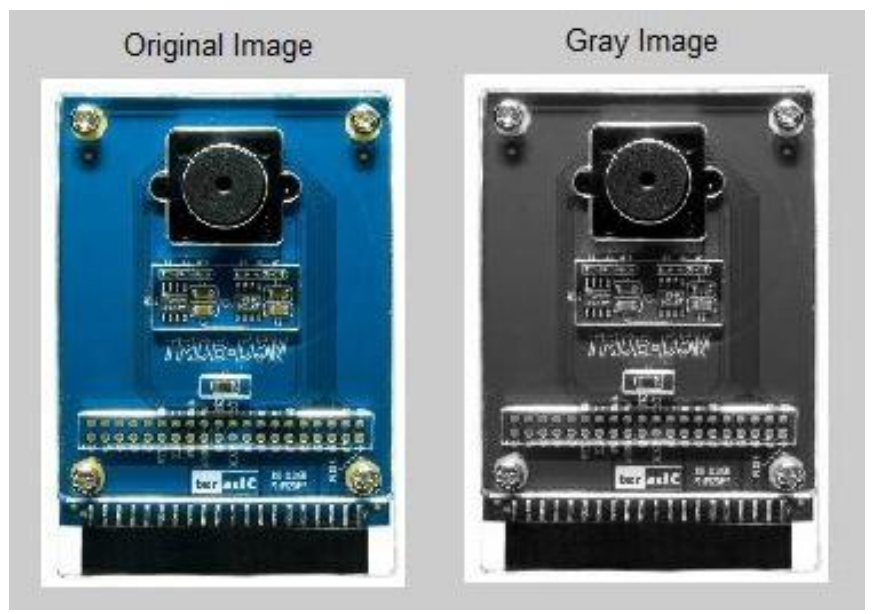

Figure 5: A] Original Image and B] Gray Image

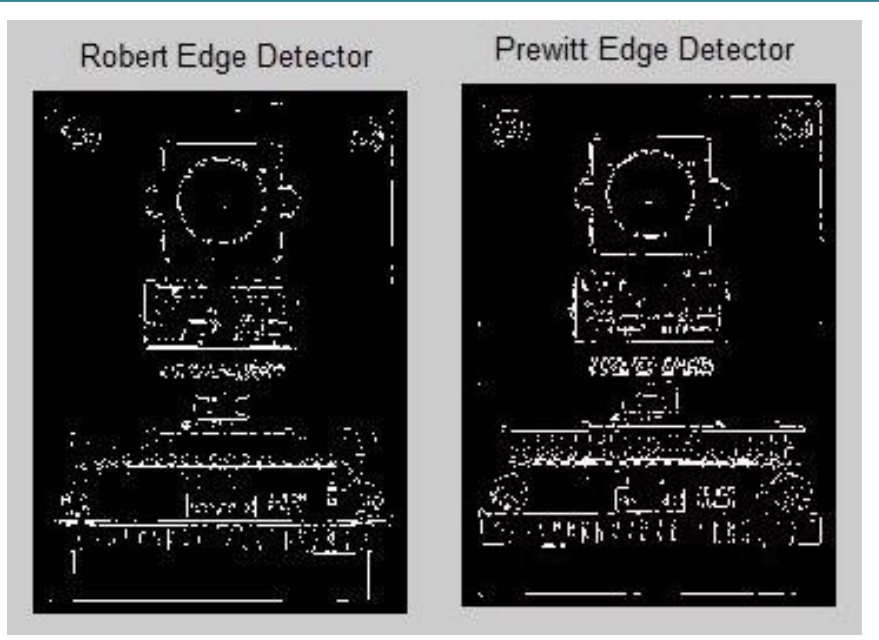

Figure 6: A] Robert Operator and B] Prewitt Operator

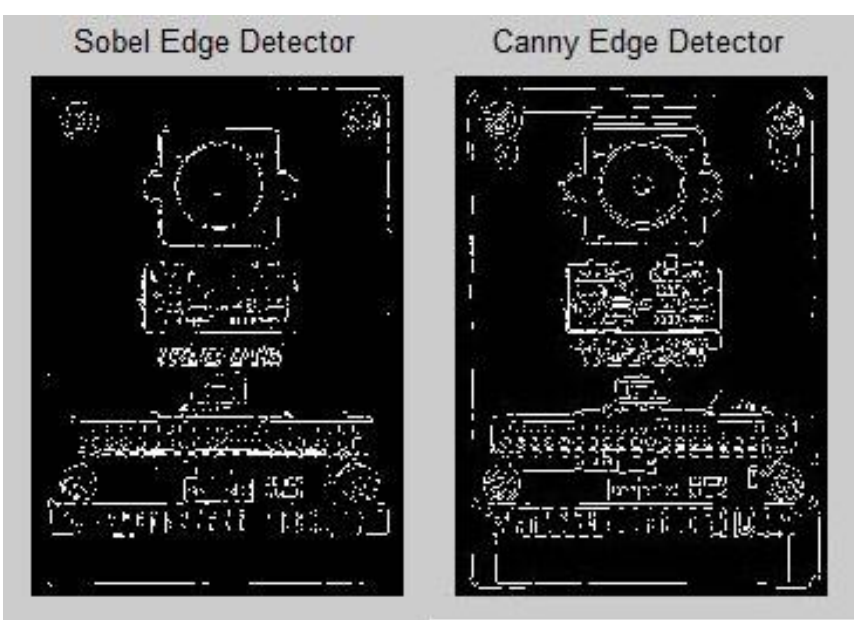

Figure 7: A] Sobel Operator and B] Canny Edge Detector

\section{Implementation of Edge Detection Algorithms}

In this paper, model based design of edge detection algorithms is discussed. Steps required to follow while implementation is given in Fig.8. Required model is designed in SIMULINK using various blocks. Using XSG, this design is converted to Xilinx System Blocks. Generated HDL file then synthesized using ISE design suit and implemented on FPGA device using JTAG programming configuration.

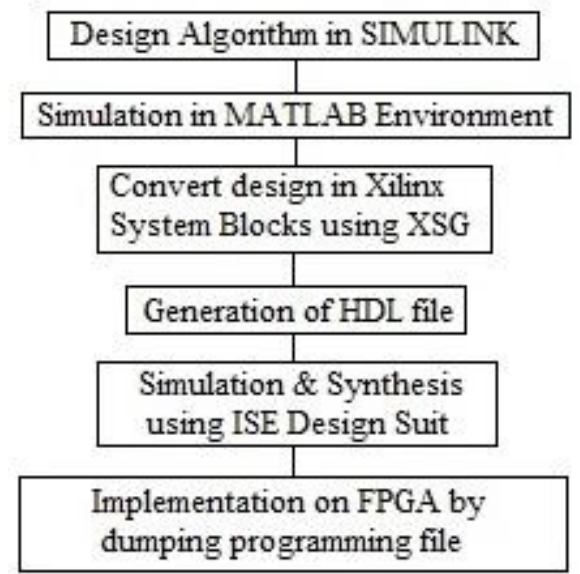

Figure 8: Steps in implementation of Canny Algorithm

\section{Volume 6 Issue 1, January 2017 www.ijsr.net}




\section{Conclusion}

Gradient based classical operators have very low latency and high throughput and are used in real time. These operators are used in several applications where accuracy is not a major issue such as surveillance, monitoring, barcode reader etc. But, in some applications (especially in the field of biomedical or machine vision) accuracy plays an important role. Thus, Canny algorithm is implemented for such applications. Computational complexity of Canny is balanced by parallel processing of FPGA which makes edge detection a real time. With the help of XSG and MATLAB/Simulink implementation process becomes much simpler than that of coding in any HDL languages. Further, texture analysis using various texture images can be done.

\section{References}

[1] R.C.Gonzalez, R.E.Woods, Digital Image Processing, 3rd edition, Prentice Hall, 2007, pp. 187-190.

[2] Raman Maini, Himanshu Aggarwal. "Study and comparison of various image edge detection techniques," Int. Journal of Image Processing. Vol. 3, pp. 1-60, Feb, 2009.

[3] J.F.Canny, "A computation approach to edge detection," IEEE Transactions on Pattern Analysis and Machine Intelligence, vol. 8, no. 6, pp. 769-798, November 1986

[4] Sangeetha D, Deepa P, "An Efficient Hardware Implementation of Canny Edge Detection Algorithm," 4th International Conference on Computer Science and Network Technology (ICCSNT 2015), pages 851-853, 2015

[5] Yao Gao-xiang, "Design of Edge Detection Algorithm for Image Sobel Based on FPGA," IEEE Transactions on Pattern Analysis and Machine Intelligence, vol. 8, no. 6, pp. 769-798, November 1986

[6] G. Bharadwaja Reddy, K. Anusudha, "Implementation of image edge detection on FPGA using XSG," International Conference on Circuit, Power and Computing Technologies (ICCPCT), 2016

[7] P. K. Dash, Shashank Pujari, Sofia Nayak, "Implementation of Edge Detection Using FPGA \& Model Based approach," ICICES2014

[8] Jeyakumar R, Prakash M, Sivanantham S, Sivasankaran $\mathrm{K}$, "FPGA Implementation of Edge Detection using Canny Algorithm," Online International Conference on Green Engineering and Technologies (IC-GET 2015), pages 1-4, 2015

[9] http://in.mathworks.com/products/simulink/

[10] Xilinx System Generator User's Guide, www.xilinx.com

\section{Author Profile}

Avinash G. Mahalle received B.Tech degree in Electronics and Telecommunication from SGGSIET, Nanded in 2014. He is now pursuing his M.Tech in Electronics System and Communication from GCOE, Amravati, Maharashtra. 